\title{
Monitoring influenza vaccine effectiveness using the national influenza surveillance system
}

Ausenda Machado (1), Maria Graça Freitas (2), Raquel Guiomar(3), Carlos Matias Dias(1), Baltazar Nunes(1)

1. Department of Epidemiology, National Health Institute Dr Ricardo Jorge, Lisbon, Portugal;

2. General Directorate For Health, Lisbon, Portugal;

3. Department of Infeccious Diseases, National Health Institute Dr Ricardo Jorge, Lisbon, Portugal

\section{Background and objectives}

Due to the annual reformulation of the influenza vaccine composition estimates of the vaccine effectiveness (VE) are required every season.

A Portuguese influenza surveillance system is in place, and data obtained by this system may be used to evaluate VE (compared to studies specifically designed to this purpose).

This study intends to evaluate the feasibility of using the national influenza surveillance system for monitoring the influenza VE (MonitorEVA). 


\section{Background \\ and objectives}

\section{Methods}

Results

Conclusions/

Main messages

\section{Methods}

Test Negative Design

(TND)

Sample of GP/ emergency room

Select ILI cases (clinical signs and symptoms)

\section{Data collection by the GP}

1. Standardized questionnaire:

2. Nasopharyngeal swab

\section{Laborayory analysis}

(RT-PCR / Culture)

CONTROLS (ILI-)

negative for any influenza virus

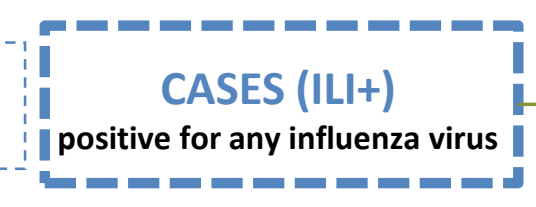

Statistical analysis

$\mathrm{VE}=1$ - OR being vaccinated in cases versus controls adjusted for confounders by logistic regression

\section{Data analysis}

Weighted by sex and age of the Portuguese population

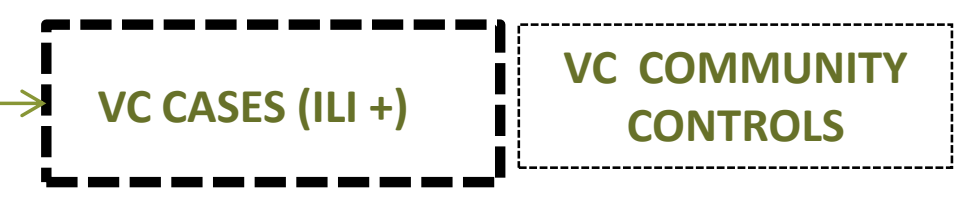

\section{Statistical analysis}

$E V=\frac{P P V-P C V}{P P V(1-P C V)} \quad \mathrm{PPV}=$ Proportion of vaccinated community PCV Proportion of vaccinated cases Farrington method to adjust for age group 


\section{Background}

and

objectives
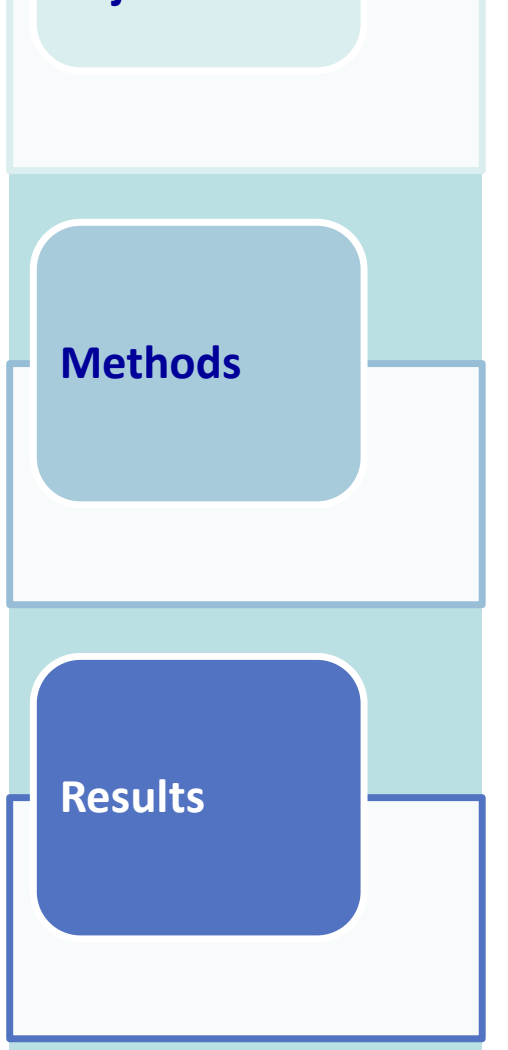

Conclusions/

Main messages

\section{Results}

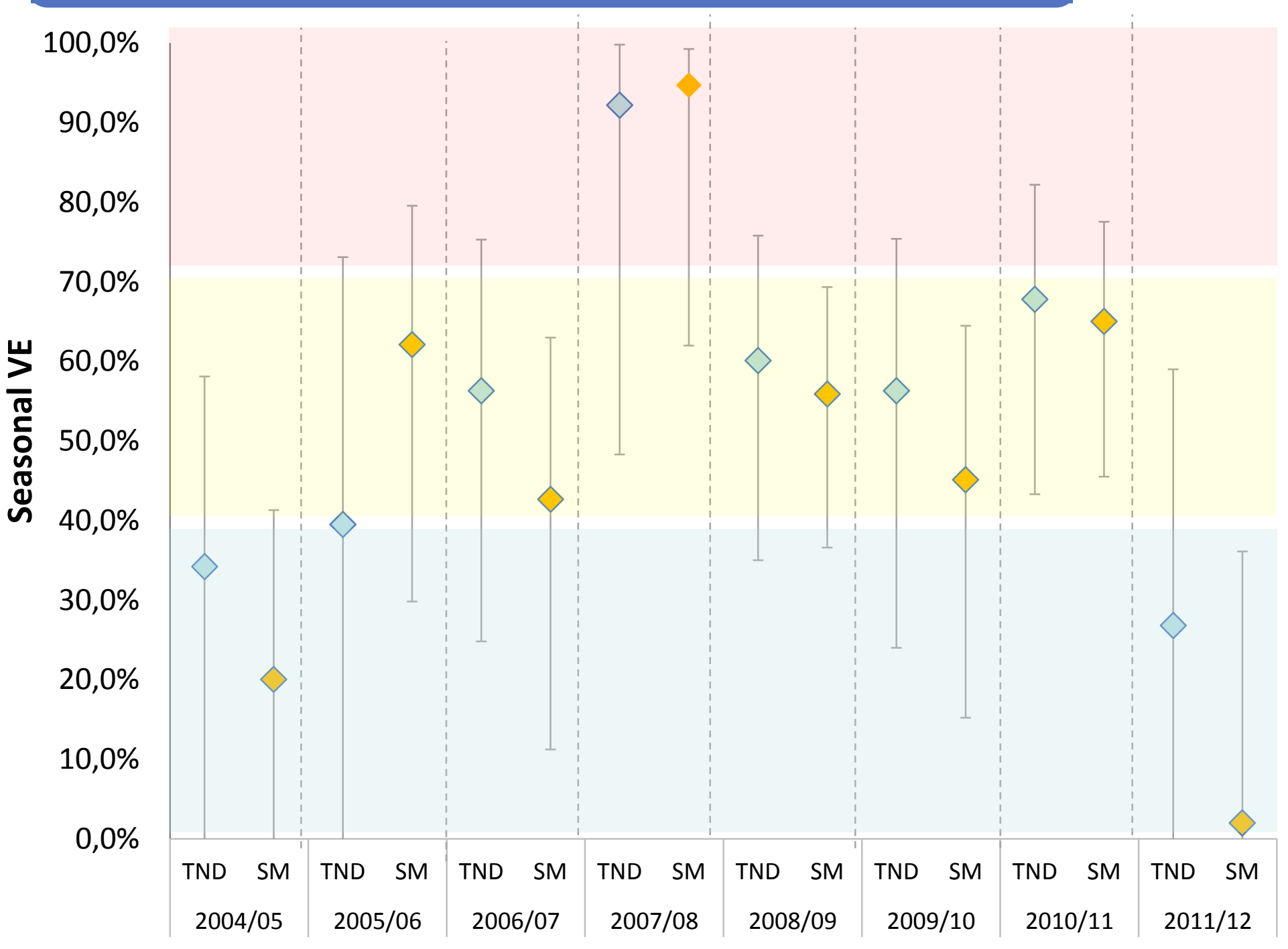

FIGURE 1. SEASONAL VACCINE EFFECTIVENESS AGAINST INFLUENZA ESTIMATED USING TND AND SM 


\section{Background}

and objectives

\section{Methods}

Results

Conclusions/

Main

messages

\section{Results}

TABLE 1. ADJUSTED VACCINE EFFECTIVENESS AGAINST INFLUENZA: COMPARISON BETWEEN MONITOREVA AND OTHER TND AD-HOC STUDIES

\section{MonitorEVA EuroEVA* IMOVE**}

$\begin{array}{lllll}2008 / 09 & V E & 23,6 & -3,0 & 59,0\end{array}$

(65 and plus)

$\mathrm{IC} 95 \% \quad(-87 ; 69) \quad(-316 ; 75) \quad(15 ; 80)$

$\begin{array}{llll}2009 / 10 & \text { VE } & 67,0 & 72,0\end{array}$

(pandemic vaccine)

\begin{tabular}{|ccccc|}
\hline \multirow{4}{*}{$2010 / 11$} & IC95\% & $(-60 ; 93)$ & & $(46 ; 86)$ \\
\hline (seasonal vaccine) & EV ajustada & 44,0 & 58,2 & 52,0 \\
\cline { 2 - 5 } & IC95\% & $(-3,6 ; 69,3)$ & $(-61 ; 89)$ & $(30 ; 67)$ \\
\hline $2011 / 12$ & EV ajustada & 5,0 & 48,8 & 25,0 \\
\cline { 2 - 5 } (seasonal vaccine) & IC95\% & $(-80,9 ; 50,2)$ & $(0 ; 74)$ & $(6 ; 47)$ \\
\hline
\end{tabular}

*EuroEVA: National study to estimate VE

**I-MOVE: European multicentric case-control study 


\section{Background}

and objectives

\section{Methods}

Results

Conclusions/

Main

messages

\section{Conclusions}

$\square \quad$ SM estimates were in accordance to the TND ones but for the majority of the SM VE was lower than the TND.

$\square \quad$ Sample size and data quality are sufficient to obtain crude VE estimates with statistical significance (if VE is higher than 50\%), however allowing less precise estimates.

$\square \quad$ The surveillance data allowed the VE monitoring indicating if the VE was higher than $70 \%$ and less than $50 \%$.

\section{Main messages}

$\square \quad$ Improvement of data quality in the surveillance program seems a potential way of improving precision and closing the gap between the two methods proposed.

$\square \quad$ Despite being less precise data from national influenza surveillance program seem to be a less expensive and adequate alternative to assess vaccine effectiveness. 